\title{
Semi-classical Linear Functionals of Class Four: The Symmetric Case
}

\author{
M. Zaatra \\ Institut Suprieur des Sciences et Techniques des Eaux de Gabs. Campus universitaire, Gabs 6072, Tunisia.
}

medzaatra@yahoo.fr

\begin{abstract}
In this paper, we obtain all the symmetric semi-classical linear functionals of class four taking into account the irreducible expression of the corresponding Pearson equation. We focus our attention on their integral representations. Thus, some linear functionals very well known in the literature, associated with perturbations of semi-classical linear functionals of class two at most, appear as well as new linear functionals which have not been studied.
\end{abstract}

Keywords: Orthogonal polynomials, Semi-classical linear functionals, Integral representations

2000 MSC No: Primary 33C45; Secondary 42C05

\section{Introduction}

Let $\mathcal{P}$ be the linear space of polynomials with complex coefficients and let $\mathcal{P}^{\prime}$ be its dual. The elements of $\mathcal{P}^{\prime}$ will be called either linear functionals or linear forms. We denote by $\langle u, f\rangle$ the action of $u \in \mathcal{P}^{\prime}$ on $f \in \mathcal{P}$. In particular, we denote by $(u)_{n}:=\left\langle u, x^{n}\right\rangle, n \geq 0$, the moments of $u$. For any linear functional $u$ and any polynomial $h$ let $D u=u^{\prime}$, $h u, \delta_{c}$, and $(x-c)^{-1} u$ be the linear functionals defined by $\left\langle u^{\prime}, f\right\rangle:=-\left\langle u, f^{\prime}\right\rangle,\langle h u, f\rangle:=\langle u, h f\rangle,\left\langle\delta_{c}, f\right\rangle:=f(c)$, and $\left\langle(x-c)^{-1} u, f\right\rangle:=\left\langle u, \theta_{c} f\right\rangle$ where $\left(\theta_{c} f\right)(x)=\frac{f(x)-f(c)}{x-c}, c \in \mathbb{C}, f \in \mathcal{P}$.

It is straightforward to prove that for $c, d \in \mathbb{C}, c \neq d, f \in \mathcal{P}$ and $u \in \mathcal{P}^{\prime}$ (see [15])

$$
\begin{gathered}
(f u)^{\prime}=f^{\prime} u+f u^{\prime}, \\
(x-c)^{-1}((x-c) u)=u-(u)_{0} \delta_{c}, \\
((x-d)(x-c))^{-1}((x-d)(x-c) u)=u+\frac{1}{c-d}\left(\left(d(u)_{0}-(u)_{1}\right) \delta_{c}-\left(c(u)_{0}-(u)_{1}\right) \delta_{d}\right) .
\end{gathered}
$$

Let us define the operator $\sigma: \mathcal{P} \rightarrow \mathcal{P}$ by $(\sigma f)(x):=f\left(x^{2}\right)$. Then, we define the even part $\sigma u$ of a linear functional $u$ by $\langle\sigma u, f\rangle=\langle u, \sigma f\rangle$.

Therefore, we have [14]

$$
\begin{gathered}
f(x) \sigma u=\sigma\left(f\left(x^{2}\right) u\right), \\
\sigma u^{\prime}=2(\sigma(x u))^{\prime} .
\end{gathered}
$$

The linear functional $u$ is said to be regular (quasi-definite) if there exists a sequence $\left\{P_{n}\right\}_{n \geq 0}$ of polynomials with $\operatorname{deg} P_{n}=n, n \geq 0$, such that

$$
\left\langle u, P_{n} P_{m}\right\rangle=r_{n} \delta_{n, m}, \quad n, m \geq 0, \quad r_{n} \neq 0, \quad n \geq 0 .
$$


We can always assume that each $P_{n}$ is monic i.e. $P_{n}(x)=x^{n}+$ lower degree terms. Then the sequence $\left\{P_{n}\right\}_{n \geq 0}$ is said to be orthogonal with respect to $u$ (MOPS in short). It is a very well known fact that the sequence $\left\{P_{n}\right\}_{n \geq 0}$ satisfies a three term recurrence relation (see, for instance, the monograph by T. S. Chihara (see [7]))

$$
\begin{aligned}
& P_{n+2}(x)=\left(x-\xi_{n+1}\right) P_{n+1}(x)-\rho_{n+1} P_{n}(x), \quad n \geq 0, \\
& P_{1}(x)=x-\xi_{0}, \quad P_{0}(x)=1,
\end{aligned}
$$

with $\left(\xi_{n}, \rho_{n+1}\right) \in \mathbb{C} \times \mathbb{C}^{*}, n \geq 0$. By convention, we set $\rho_{0}=(u)_{0}=1$.

A linear functional $u$ is called symmetric if $(u)_{2 n+1}=0, n \geq 0$. The conditions $(u)_{2 n+1}=0, n \geq 0$, are equivalent to the fact that the corresponding MOPS, $\left\{P_{n}\right\}_{n \geq 0}$, satisfies the recurrence relation $(6)$ with $\xi_{n}=0, n \geq 0$ $($ see $[7])$.

A regular linear functional $u$ is said to be positive definite if $\langle u, f\rangle>0$ for all $f \in \mathcal{P}$ such that $f(x) \geq 0$, for every $x \in \mathbb{R}$ and $f \neq 0$ or, equivalently, its MOPS satisfies (6) with $\xi_{n} \in \mathbb{R}$ and $\rho_{n} \in \mathbb{R}_{+}^{*}$ for all $n \geq 1$ (see $[15]$ ).

From a structural point of view, a very important family of regular linear functionals has been exhaustively analyzed in the literature during the last two decades. Let us recall that a linear functional $\tilde{u}$ is said to be semiclassical when it is regular and there exist two polynomials $\tilde{\Phi}$, a monic polynomial, and $\tilde{\Psi}$, deg $\tilde{\Psi} \geq 1$, such that

$$
(\tilde{\Phi} \tilde{u})^{\prime}+\tilde{\Psi} \tilde{u}=0
$$

This is the Pearson equation associated with the linear functional. The class of the semi-classical linear functional $\tilde{u}$ is defined as the nonnegative number $\tilde{s}=\max (\operatorname{deg} \tilde{\Psi}-1, \operatorname{deg} \tilde{\Phi}-2)$ if and only if the following condition is satisfied

$$
\prod_{c}\left(\left|\tilde{\Phi}^{\prime}(c)+\tilde{\Psi}(c)\right|+\left|\left\langle\tilde{u}, \theta_{c} \tilde{\Psi}+\theta_{c}^{2} \tilde{\Phi}\right\rangle\right|\right)>0,
$$

where $c$ belongs to the set of zeros of $\tilde{\Phi}$. Notice that this condition means that the polynomials $\tilde{\Phi}$ and $\tilde{\Phi}^{\prime}(x)+\tilde{\Psi}(x)$ are coprime as well as the equation (7) is irreducible (see [15]).

The corresponding MOPS $\left\{P_{n}\right\}_{n \geq 0}$ is said to be semi-classical of class $\tilde{s}$. When $\tilde{s}=0, \tilde{u}$ is a classical linear functional (Hermite, Laguerre, Jacobi, and Bessel). See [7] and [15].

Semi-classical linear functionals associated with weight functions were considered first by J. Shohat [18] in the framework of the existence of sequences of orthogonal polynomials satisfying second order linear differential equations with polynomial coefficients (holonomic equations). Later on, P. Maroni and coworkers have extensively studied such a kind of linear functionals with a special emphasis on their structure properties. For instance, [15] constitutes a relevant survey on the subject. Many examples appearing in the literature, mainly related to spectral perturbations of classical linear functionals, are semi-classical but a constructive theory of such linear functionals remains open.

Indeed, the classification of semi-classical linear functionals according to some criteria of optimal information from their Pearson equation, plays a central role in the constructive theory of such linear functionals. In [5] S. Belmehdi makes use of this approach to provide a full description of all semi-classical linear functionals of class $s=1$. In [1] the classification of symmetric semi-classical linear functionals of such a class is given. Recently, the semi-classical linear functionals of class $s=2$ are completely described by F. Marcellán et al. in [8,10]. In [9], the authors gives a complete description of all symmetric semi-classical linear of class $s=3$ using such an approach. Finally, a complete study of the class of the symmetric companion $u$ of a linear functional $v$ in terms of the class of $v$ is done in [4]. Notice that some examples of symmetric semi-classical linear functionals of class greater than 2 have been analyzed in [2]. The aim of our contribution is to cover this gap.

As a first step, it is natural to deal with the description of the symmetric semi-classical linear functionals of class $s=4$. 
The structure of the manuscript is as follows. In Section 2, the irreducible canonical Pearson equations associated with symmetric semi-classical linear functionals of class $s=4$ are obtained. Thus, fifteen irreducible canonical cases appear. In Section 3, the integral representation of such linear functionals is given, with a special emphasis on the positive definite case.

\section{Irreducible canonical functional equations}

First of all, let us recall the following result:

Proposition 2.1. [1] Let $u$ be a symmetric semi-classical linear functional of class s, satisfying (8). If $s$ is an even nonnegative integer number, then $\Phi$ is an even polynomial function and $\Psi$ is an odd polynomial function. If $s$ is an odd nonnegative integer number, then $\Phi$ is an odd polynomial function and $\Psi$ is an even polynomial function.

In the sequel, we will assume that the linear functional $\tilde{u}$ is symmetric and semi-classical of class $\tilde{s}=4$. Then, according to the above proposition $\tilde{u}$ satisfies (7) with

$$
\tilde{\Phi}(x)=\tilde{c}_{6} x^{6}+\tilde{c}_{4} x^{4}+\tilde{c}_{2} x^{2}+\tilde{c}_{0}, \tilde{\Psi}(x)=\tilde{a}_{5} x^{5}+\tilde{a}_{3} x^{3}+\tilde{a}_{1} x,\left|\tilde{c}_{6}\right|+\left|\tilde{a}_{5}\right| \neq 0 .
$$

As a consequence, the moments $(\tilde{u})_{n}$ of the linear functional $\tilde{u}$ satisfy the linear difference equation

$$
\left(\tilde{a}_{5}-(2 n+1) \tilde{c}_{6}\right)(\tilde{u})_{2 n+6}+\left(\tilde{a}_{3}-(2 n+1) \tilde{c}_{4}\right)(\tilde{u})_{2 n+4}+\left(\tilde{a}_{1}-(2 n+1) \tilde{c}_{2}\right)(\tilde{u})_{2 n+2}-(2 n+1) \tilde{c}_{0}(\tilde{u})_{2 n}=0, n \geq 0,
$$

with $(\tilde{u})_{0}=1$ and $(\tilde{u})_{2 n+1}=0, n \geq 0$. Then, the set of solutions is a linear space of dimension at most three.

The linear functional $h_{a} \tilde{u}$ (dilation of $\tilde{u}$ ) is defined by

$$
\left\langle h_{a} \tilde{u}, f\right\rangle:=\left\langle\tilde{u}, h_{a} f\right\rangle:=\langle\tilde{u}, f(a x)\rangle, f \in \mathcal{P} .
$$

The semi-classical character of a linear functional is preserved by a dilation. Indeed, the dilated linear functional $u=\left(h_{a^{-1}}\right) \tilde{u}, a \in \mathbb{C}^{*}$, satisfies

$$
(\Phi u)^{\prime}+\Psi u=0
$$

with

$$
\Phi(x)=a^{-t} \tilde{\Phi}(a x), \quad \Psi(x)=a^{1-t} \tilde{\Psi}(a x), \quad t=\operatorname{deg} \tilde{\Phi} .
$$

The sequence $\left\{\hat{P}_{n}(x)\right\}_{n \geq 0}$, where $\hat{P}_{n}(x)=a^{-n} P_{n}(a x)$, is orthogonal with respect to $u$ and fulfils $(6)$ with

$$
\hat{\xi}_{n}=0, \quad \hat{\rho}_{n+1}=\frac{\rho_{n+1}}{a^{2}}, \quad n \geq 0 .
$$

As we can see, a dilation $h_{a}$ does not modify the nature of a symmetric semi-classical linear functional. This process will be applied to the Pearson equation satisfied by a symmetric semi-classical linear functional of class $s=4$. In the sequel, $a \in \mathbb{C}^{*}$ will denote an arbitrary complex number. A convenient choice of $a$, according to the expression of $\tilde{\Phi}$, allows us to re-locate the zeros of $\tilde{\Phi}$ in the complex plane. In this way, (9) can be reduced either to some situations which appear in the literature or yield new linear functionals not yet studied. Thus we get canonical distributional equations of class four in a simple way, that becomes a pattern for the family of equations which can be reduced using a shifting.

According to Proposition 2.1, we will analyze two situations.

A. $\operatorname{deg} \tilde{\Phi}=6$ and $1 \leq \operatorname{deg} \tilde{\Psi} \leq 5$.

B. $\operatorname{deg} \tilde{\Psi}=5$ and $0 \leq \operatorname{deg} \tilde{\Phi} \leq 4$.

Case A. Set

$$
\begin{gathered}
\tilde{\Phi}(x)=\prod_{i=1}^{3}\left(x^{2}-\alpha_{i}^{2}\right), \\
\tilde{\Psi}=x\left(\tilde{a}_{4} x^{4}+\tilde{a}_{2} x^{2}+\tilde{a}_{0}\right), \quad\left|\tilde{a}_{4}\right|+\left|\tilde{a}_{2}\right|+\left|\tilde{a}_{0}\right| \neq 0 .
\end{gathered}
$$


Then $u$ satisfies

$$
\left(\prod_{i=1}^{3}\left(x^{2}-\alpha_{i}^{2} a^{-2}\right) u\right)^{\prime}+x\left(\tilde{a}_{4} x^{4}+a^{-2} \tilde{a}_{2} x^{2}+a^{-4} \tilde{a}_{0}\right) u=0 .
$$

We will discuss the following cases.

$\mathbf{A}_{1} \cdot \tilde{\Phi}$ has six simple zeros.

We choose $a$ such that $\alpha_{1}=a \neq 0, \alpha_{2}=a c$ and $\alpha_{3}=a d$ with $c^{2}, d^{2} \notin\{0,1\}$ and $c^{2} \neq d^{2}$. Thus (13) reduces to

$$
\left(\left(x^{2}-1\right)\left(x^{2}-c^{2}\right)\left(x^{2}-d^{2}\right) u\right)^{\prime}+x\left(a_{4} x^{4}+a_{2} x^{2}+a_{0}\right) u=0 .
$$

The rational function $-\frac{\Phi^{\prime}+\Psi}{\Phi^{\prime}}$ has six simple poles $1,-1, c,-c, d$ and $-d$. We denote by

$$
\alpha=-\frac{\Phi^{\prime}(1)+\Psi(1)}{\Phi^{\prime}(1)}, \beta=-\frac{\Phi^{\prime}(c)+\Psi(c)}{\Phi^{\prime}(c)} \text { and } \gamma=-\frac{\Phi^{\prime}(d)+\Psi(d)}{\Phi^{\prime}(d)}
$$

the corresponding residues. Then, after some straightforward calculation, we obtain

$$
\left(\begin{array}{l}
a_{4} \\
a_{2} \\
a_{0}
\end{array}\right)=M\left(\begin{array}{c}
2 \alpha+2 \\
2 \beta+2 \\
2 \gamma+2
\end{array}\right)
$$

where

$$
M=\left(\begin{array}{ccc}
-1 & -1 & -1 \\
c^{2}+d^{2} & d^{2}+1 & c^{2}+1 \\
-c^{2} d^{2} & -d^{2} & -c^{2}
\end{array}\right)
$$

Notice that $\operatorname{det} M=\left(c^{2}-1\right)\left(d^{2}-1\right)\left(c^{2}-d^{2}\right) \neq 0$. This means that this change of parameters is bijective. (We have the same kind of relation between the new parameters and the old ones in the other cases that we will study in the sequel.)

Now, changing the parameters in (14), according to the condition of irreducibility (8) we get

$$
\left\{\begin{array}{l}
\left(\left(x^{2}-1\right)\left(x^{2}-c^{2}\right)\left(x^{2}-d^{2}\right) u\right)^{\prime}+x\left(-2(\alpha+\beta+\gamma+3) x^{4}+2(\beta+\gamma+2\right. \\
\left.\left.+c^{2}(\alpha+\gamma+2)+d^{2}(\alpha+\beta+2)\right) x^{2}-2\left(c^{2} d^{2}(\alpha+1)+c^{2}(\gamma+1)+d^{2}(\beta+1)\right)\right) u=0, \\
\left\{|\beta|+\mid-(2 \alpha+2 \beta+2 \gamma+5)(u)_{4}+\left(2 \beta+2 \gamma+3+(2 \alpha+2 \beta+3) d^{2}-2 \beta c^{2}\right)(u)_{2}\right. \\
\left.+2 \beta c^{2}\left(d^{2}+1-c^{2}\right)-(2 \beta+1) d^{2} \mid\right\} \times\left\{|\alpha|+\mid-(2 \alpha+2 \beta+2 \gamma+5)(u)_{4}\right. \\
\left.+\left((2 \alpha+2 \gamma+3) c^{2}+(2 \alpha+2 \beta+3) d^{2}-2 \alpha\right)(u)_{2}+2 \alpha\left(c^{2}+d^{2}-1\right)-(2 \alpha+1) c^{2} d^{2} \mid\right\} \\
\times\left\{|\gamma|+\mid-(2 \alpha+2 \beta+2 \gamma+5)(u)_{4}+\left((2 \alpha+2 \gamma+3) c^{2}+2 \beta+2 \gamma+3-2 \gamma d^{2}\right)(u)_{2}\right. \\
\left.+2 \alpha d^{2}\left(c^{2}+1-d^{2}\right)-(2 \gamma+1) c^{2} \mid\right\} \neq 0 .
\end{array}\right.
$$

This is the irreducible Pearson equation satisfied by a linear functional of class four when $\Phi$ in (10) has six simple zeros.

We will proceed in a similar way in the cases listed in below.

$\mathbf{A}_{2}$. $\tilde{\Phi}$ has five different zeros and one of them is double zeros. In such a situation $\alpha_{1}=0$ and $\alpha_{2} \alpha_{3} \neq 0$.

We choose $a$ such that $\alpha_{2}=a$ and $\alpha_{3}=a c$ with $c \notin\{-1,0,1\}$. Then (13) can be written as

$$
\left(x^{2}\left(x^{2}-1\right)\left(x^{2}-c^{2}\right) u\right)^{\prime}+x\left(a_{4} x^{4}+a_{2} x^{2}+a_{0}\right) u=0 \text {. }
$$


By an appropriate choice of the coefficients $a_{4}, a_{2}$, and $a_{0},(16)$ becomes

$$
\left\{\begin{array}{l}
\left(x^{2}\left(x^{2}-1\right)^{2}\left(x^{2}-c^{2}\right) u\right)^{\prime}+x\left(-(\alpha+2 \beta+2 \gamma+6) x^{4}+\left((\alpha+2 \beta+4) c^{2}\right.\right. \\
\left.\quad+\alpha+2 \gamma+4) x^{2}-(\alpha+2) c^{2}\right) u=0, \\
\left\{-(\alpha+2 \beta+2 \gamma+5)(u)_{4}+\left((\alpha+2 \beta+3) c^{2}+\alpha+2 \gamma+3\right)(u)_{2}-(\alpha+1) c^{2}\right\} \\
\times\left\{|\beta|+\left|-(\alpha+2 \beta+2 \gamma+5)(u)_{4}+\left((\alpha+2 \beta+3) c^{2}-2 \beta\right)(u)_{2}+2 \beta\left(c^{2}-1\right)\right|\right\} \\
\times\left\{|\gamma|+\left|-(\alpha+2 \beta+2 \gamma+5)(u)_{4}+\left(\alpha+3+2 \gamma\left(1-c^{2}\right)\right)(u)_{2}+2 \gamma c^{2}\left(1-c^{2}\right)\right|\right\} \neq 0 .
\end{array}\right.
$$

This is the corresponding irreducible Pearson equation of a linear functional of class four when $\Phi$ in (10) has five different zeros and one of them is of multiplicity two.

Remark 1. If $c^{2}=-1$ and $\alpha=-2$, then we obtain the result given in [3]

$\mathbf{A}_{3} . \tilde{\Phi}$ has four different zeros and two of them are a double zero.

We choose $a$ such that $\alpha_{1}=\alpha_{2}=a$ and $\alpha_{3}=a c$ with $c \notin\{-1,0,1\}$. Then (13) becomes

$$
\left(\left(x^{2}-1\right)^{2}\left(x^{2}-c^{2}\right) u\right)^{\prime}+x\left(a_{4} x^{4}+a_{2} x^{2}+a_{0}\right) u=0 \text {. }
$$

From a suitable choice of the coefficients $a_{4}, a_{2}$, and $a_{0}$, (18) yields

$$
\left\{\begin{array}{l}
\left(\left(x^{2}-1\right)^{2}\left(x^{2}-c^{2}\right) u\right)^{\prime}+2 x\left(-(\alpha+\gamma+3) x^{4}+\left((\alpha+2) c^{2}+\alpha+\beta+2 \gamma+4\right) x^{2}\right. \\
\left.\quad-\gamma-1-(\alpha+\beta+2) c^{2}\right) u=0, \\
\left\{|\beta|+\left|-(2 \alpha+2 \gamma+5)(u)_{4}+\left((2 \alpha+3) c^{2}+2 \beta+2 \gamma+3\right)(u)_{2}+2 \beta-(2 \beta+1) c^{2}\right|\right\} \\
\quad \times\left\{|\gamma|+\left|-(2 \alpha+2 \gamma+5)(u)_{4}+2\left(3+\alpha+\beta+\gamma\left(2-c^{2}\right)\right)(u)_{2}+2 \gamma c^{2}\left(1-c^{2}\right)-2 \gamma-1\right|\right\} \neq 0 .
\end{array}\right.
$$

This is an irreducible Pearson equation of a linear functional of class four, when $\Phi$ in (10) has four different zeros when two of them are of multiplicity two.

$\mathbf{A}_{4} \cdot \tilde{\Phi}$ has three different zeros of multiplicity two, i.e. $\alpha_{1}=0$ and $\alpha_{2} \alpha_{3} \neq 0$.

We choose $a$ such that $\alpha_{2}=\alpha_{3}=a$.

(13) becomes

$$
\left(x^{2}\left(x^{2}-1\right)^{2} u\right)^{\prime}+x\left(a_{4} x^{4}+a_{2} x^{2}+a_{0}\right) u=0 .
$$

By a skilful choice of the coefficients $a_{4}, a_{2}$ and $a_{0},(20)$ can be written as

$$
\left\{\begin{array}{l}
\left(x^{2}\left(x^{2}-1\right)^{2} u\right)^{\prime}+x\left(-(2 \alpha+2 \beta+7) x^{4}+2(2 \alpha+\beta+\gamma+5) x^{2}-2 \alpha-3\right) u=0, \\
\left\{-(\alpha+\beta+3)(u)_{4}+(2 \alpha+\beta+\gamma+4)(u)_{2}-\alpha-1\right\} \\
\times\left\{|\gamma|+\left|-(\alpha+\beta+3)(u)_{4}+(\alpha+\gamma+2)(u)_{2}+\gamma\right|\right\} \neq 0 .
\end{array}\right.
$$

This is the irreducible Pearson equation for a functional of class four, when $\Phi$ in (10) has a three different zeros of multiplicity two.

$\mathbf{A}_{5}$. $\tilde{\Phi}$ has three different zeros and one of them of multiplicity four. This means that either $\alpha_{1}=\alpha_{2}=0$ and $\alpha_{3} \neq 0$.

We choose $a$ such that $\alpha_{3}=a$. (13) can be written as

$$
\left(x^{4}\left(x^{2}-1\right) u\right)^{\prime}+x\left(a_{4} x^{4}+a_{2} x^{2}+a_{0}\right) u=0 .
$$

By an appropriate choice of the coefficients $a_{4}, a_{2}$ and $a_{0},(22)$ reads as

$$
\left\{\begin{array}{l}
\left(x^{4}\left(x^{2}-1\right) u\right)^{\prime}+x\left(-(\alpha+2 \gamma+6) x^{4}+(\alpha-2 \beta+4) x^{2}+2 \beta\right) u=0, \\
\left\{-(\alpha+2 \gamma+5)(u)_{4}+(\alpha-2 \beta+3)(u)_{2}+2 \beta\right\} \\
\times\left\{|\gamma|+\left|-(\alpha+2 \gamma+5)(u)_{4}-2(\beta+\gamma)(u)_{2}-2 \gamma\right|\right\} \neq 0 .
\end{array}\right.
$$


This is the irreducible Pearson equation for a functional of class four, when $\Phi$ in (10) has a three different zeros: two of them are simple and the other one has multiplicity four.

$\mathbf{A}_{6} . \tilde{\Phi}$ has two different zeros of multiplicity three.

We choose $a$ such that $\alpha_{1}=\alpha_{2}=\alpha_{3}=a$. Then (13) becomes

$$
\left(\left(x^{2}-1\right)^{3} u\right)^{\prime}+x\left(a_{4} x^{4}+a_{2} x^{2}+a_{0}\right) u=0 .
$$

From a suitable choice of the coefficients $a_{4}, a_{2}$ and $a_{0},(24)$ can be written as

$$
\left\{\begin{array}{l}
\left(\left(x^{2}-1\right)^{3} u\right)^{\prime}+2 x\left(-(\alpha+3) x^{4}+2(\alpha+3) x^{2}-\alpha-2 \beta-3\right) u=0 \\
\left.|\beta|+\left|-(2 \alpha+5)(u)_{4}+2(\alpha+3)(u)_{2}-4 \beta-1\right|\right\} \neq 0 .
\end{array}\right.
$$

This is the irreducible Pearson equation for a functional of class four, when $\Phi$ in (10) has a two zeros of multiplicity three.

A $7 . \tilde{\Phi}$ has one zero of multiplicity six, i.e. $\alpha_{1}=\alpha_{2}=\alpha_{3}=0$.

(13) becomes

$$
\left(x^{6} u\right)^{\prime}+x\left(\tilde{a}_{4} x^{4}+a^{-2} \frac{\tilde{\Psi}^{\prime \prime \prime}(0)}{6} x^{2}+a^{-4} \tilde{\Psi}^{\prime}(0)\right) u=0 .
$$

We can consider two subcases.

$\mathbf{A}_{7.1} \cdot \tilde{\Psi}^{\prime}(0) \neq 0$.

Let $a$ be such that $a^{-4} \tilde{a}_{0}=-8$. Then (26) reduces to

$$
\left(x^{6} u\right)^{\prime}+x\left(a_{4} x^{4}+a_{2} x^{2}-8\right) u=0 .
$$

By a skilful choice of the coefficients $a_{4}$ and $a_{2},(27)$ yields

$$
\left\{\begin{array}{l}
\left(x^{6} u\right)^{\prime}+x\left(-(2 \alpha+1) x^{4}-2 \beta x^{2}-8\right) u=0 \\
\alpha(u)_{4}+\beta(u)_{2}+4 \neq 0
\end{array}\right.
$$

This is the irreducible Pearson equation for a functional of class four, when $\Phi$ in (10) has a zero of multiplicity six and this zero is a simple zero of $\Psi$.

$\mathbf{A}_{7.2} \cdot \tilde{\Psi}^{\prime}(0)=0$ and $\tilde{\Psi}^{\prime \prime \prime}(0) \neq 0$.

If we chose $a$ such that $a^{-2} \tilde{\Psi}^{\prime \prime \prime}(0)=-24$, then we obtain

$$
\left(x^{6} u\right)^{\prime}+x^{3}\left(a_{4} x^{2}-4\right) u=0 \text {. }
$$

By an appropriate choice of the coefficient $a_{4},(29)$ reads

$$
\left\{\begin{array}{l}
\left(x^{6} u\right)^{\prime}-x^{3}\left((4 \alpha-1) x^{2}+4\right) u=0 \\
(2 \alpha-1)(u)_{4}+2(u)_{2} \neq 0
\end{array}\right.
$$

This is the irreducible Pearson equation for a functional of class four, when $\Phi$ in (10) has a zero of multiplicity six and it is a triple zero of $\Psi$.

Remark 2. (1) If zero is a root of multiplicity five of $\tilde{\Psi}$, the equation (26) writes

$$
\left(x^{6} u\right)^{\prime}+\tilde{a}_{4} x^{5} u=0 .
$$

Then, we obtain

$$
\left(2 n+1-\tilde{a}_{4}\right)(u)_{2 n+6}=0, \quad n \geq 0 .
$$

So after a certain rank the Hankel determinants associated with $u$ are all hopeless, by following $u$ is not regular. Then the case is to reject. 
(2) From (30), we have

$$
x^{2} u=k v,
$$

where $k$ is a normalization term and $v$ is the symmetric semi-classical linear form of class two defined by the Pearson equation (see $[8,10,17])$

$$
\left\{\begin{array}{l}
\left(x^{4} v\right)^{\prime}-x\left((4 \alpha-1) x^{2}+4\right) v=0, \\
(2 \alpha+1)(v)_{2}+2 \neq 0 .
\end{array}\right.
$$

The linear functional $u$ defined by (31) is regular if and only if (see [12])

$$
\left\langle u, P_{n+1}^{(\alpha)}\right\rangle\left\langle u, x P_{n}^{(\alpha)}\right\rangle-\left\langle u, P_{n}^{(\alpha)}\right\rangle\left\langle u, x P_{n+1}^{(\alpha)}\right\rangle \neq 0, n \geq 0,
$$

where $P_{n}^{(\alpha)}(x), n \geq 0$, are the monic polynomials orthogonal with to $v$.

Case B. Set

$$
\begin{gathered}
\tilde{\Phi}(x)=\tilde{c}_{4} x^{4}+\tilde{c}_{2} x^{2}+c_{0}, \quad\left|\tilde{c}_{4}\right|+\left|\tilde{c}_{2}\right|+\left|\tilde{c}_{0}\right| \neq 0, \\
\tilde{\Psi}=x\left(\tilde{a}_{4} x^{4}+\tilde{a}_{2} x^{2}+\tilde{a}_{0}\right), \quad \tilde{a}_{4} \neq 0 .
\end{gathered}
$$

We will analyze the following situations:

$\mathbf{B}_{1} . \tilde{\Phi}$ has four simple zeros $\alpha_{1},-\alpha_{1}, \alpha_{2}$ and $-\alpha_{2}$.

$\tilde{u}$ and $u$ satisfy, respectively,

$$
\begin{gathered}
\left(\prod_{i=1}^{2}\left(x^{2}-\alpha_{i}^{2}\right) \tilde{u}\right)^{\prime}+x\left(\tilde{a}_{4} x^{4}+\tilde{a}_{2} x^{2}+\tilde{a}_{0}\right) \tilde{u}=0, \\
\left(\prod_{i=1}^{2}\left(x^{2}-\alpha_{i}^{2} a^{-2}\right) u\right)^{\prime}+x\left(\tilde{a}_{4} a^{2} x^{4}+\tilde{a}_{2} x^{2}+a^{-2} \tilde{a}_{0}\right) u=0 .
\end{gathered}
$$

Let $a$ be such that $\alpha_{1}=a$ and $\alpha_{2}=a c$ with $c \notin\{-1,0,1\}$. Then (32) becomes

$$
\left(\left(x^{2}-1\right)\left(x^{2}-c^{2}\right) u\right)^{\prime}+x\left(a_{4} x^{4}+a_{2} x^{2}+a_{0}\right) u=0 .
$$

By an appropriate choice of the coefficients $a_{4}, a_{2}$, and $a_{0}$, with $\lambda \neq 0$ we get

$$
\left\{\begin{array}{l}
\left(\left(x^{2}-1\right)\left(x^{2}-c^{2}\right) u\right)^{\prime}+2 x\left(\lambda x^{4}-\left(\lambda\left(c^{2}+1\right)+\alpha+\beta+2\right) x^{2}\right. \\
\left.\quad+(\lambda+\alpha+1) c^{2}+\beta+1\right) u=0 \\
\left\{|\beta|+\left|2 \lambda(u)_{4}-(2 \lambda+2 \alpha+2 \beta+3)(u)_{2}+2 \beta\left(1-c^{2}\right)+1\right|\right\} \\
\quad \times\left\{|\alpha|+\left|2 \lambda(u)_{4}-\left(2 \lambda c^{2}+2 \alpha+2 \beta+3\right)(u)_{2}+(2 \alpha+1) c^{2}-2 \alpha\right|\right\} \neq 0 .
\end{array}\right.
$$

This is the irreducible Pearson equation for a linear functional of class four, when $\Phi$ in (10) has four simple zeros.

$\mathbf{B}_{2} . \tilde{\Phi}$ has three simple zeros and one of them of multiplicity two. This means $\alpha_{1}=0$ and $\alpha_{2} \neq 0$.

We choose $a$ in such a way that $\alpha_{2}=a$. Then (32) reads as

$$
\left(x^{2}\left(x^{2}-1\right) u\right)^{\prime}+x\left(a_{4} x^{4}+a_{2} x^{2}+a^{-2} a_{0}\right) u=0 .
$$

By a suitable choice of the coefficients $a_{4}, a_{2}$ and $a_{0}$, with $\lambda \neq 0$ we obtain

$$
\left\{\begin{array}{l}
\left(x^{2}\left(x^{2}-1\right) u\right)^{\prime}+x\left(2 \lambda x^{4}-(2 \lambda+\alpha+2 \beta+4) x^{2}+\alpha+2\right) u=0, \\
\left\{2 \lambda(u)_{4}-(2 \lambda+\alpha+2 \beta+3)(u)_{2}+\alpha+1\right\} \\
\times\left\{|\beta|+\left|2 \lambda(u)_{4}-(\alpha+2 \beta+3)(u)_{2}-2 \beta\right|\right\} \neq 0 .
\end{array}\right.
$$


This is the irreducible Pearson equation for a linear functional of class four, when $\Phi$ in (10) has a zero of multiplicity two.

$\mathbf{B}_{3} . \tilde{\Phi}$ has two zeros of multiplicity two.

We choose $a$ such that $\alpha_{1}=\alpha_{2}=a$. (32) becomes

$$
\left(\left(x^{2}-1\right)^{2} u\right)^{\prime}+x\left(a_{4} x^{4}+a_{2} x^{2}+a^{-2} a_{0}\right) u=0 .
$$

By an appropriate choice of the coefficients $a_{4}, a_{2}$ and $a_{0}$ with $\lambda \neq 0,(37)$ yields

$$
\left\{\begin{array}{l}
\left(\left(x^{2}-1\right)^{2} u\right)^{\prime}+2 x\left(\lambda x^{4}-(2 \lambda+\alpha+2) x^{2}+\lambda+\alpha+\beta+2\right) u=0, \\
|\beta|+\left|2 \lambda(u)_{4}-(2 \lambda+2 \alpha+3)(u)_{2}+2 \beta+1\right| \neq 0 .
\end{array}\right.
$$

This is the irreducible Pearson equation for a linear functional of class four, when $\Phi$ in (10) has two zeros of multiplicity two.

$\mathbf{B}_{4} . \tilde{\Phi}$ has one zero of multiplicity four $\alpha_{1}=\alpha_{2}=0$.

We choose $a$ in such a way that $\tilde{a}_{4} a^{2}=2$. Then (32) reads as

$$
\left(x^{4} u\right)^{\prime}+x\left(2 x^{4}+a_{2} x^{2}+a^{-2} a_{0}\right) u=0 .
$$

By a suitable choice of the coefficients $a_{2}$ and $a_{0}$, (39) can be written as

$$
\left\{\begin{array}{l}
\left(x^{4} u\right)^{\prime}+2 x\left(x^{4}-(\alpha+2) x^{2}-\beta\right) u=0, \\
2(u)_{4}-(2 \alpha+3)(u)_{2}-2 \beta \neq 0 .
\end{array}\right.
$$

This is the irreducible Pearson equation for a linear functional of class four, when $\Phi$ in (10) has a zero of multiplicity four.

$\mathbf{B}_{5} . \tilde{\Phi}(x)=x^{2}-\alpha_{1}^{2}$.

We need to discuss the following situations.

$\mathbf{B}_{5.1} . \tilde{\Phi}$ has two simple zeros.

$\tilde{u}$ and $u$ satisfy, respectively,

$$
\begin{gathered}
\left(\left(x^{2}-\alpha_{1}^{2}\right) \tilde{u}\right)^{\prime}+x\left(\tilde{a}_{4} x^{4}+\tilde{a}_{2} x^{2}+\tilde{a}_{0}\right) \tilde{u}=0, \\
\left(\left(x^{2}-\alpha_{1}^{2} a^{-2}\right) u\right)^{\prime}+x\left(a^{4} \tilde{a}_{4} x^{4}+a^{2} \tilde{a}_{2} x^{2}+\tilde{a}_{0}\right) u=0 .
\end{gathered}
$$

If we choose $a$ such that $\alpha_{1}=a$, then we have

$$
\left(\left(x^{2}-1\right) u\right)^{\prime}+x\left(a_{4} x^{4}+a_{2} x^{2}+a_{0}\right) u=0 .
$$

By an appropriate choice of the coefficients $a_{4}, a_{2}$ and $a_{0}$ with $\lambda \neq 0,(42)$ reads as

$$
\left\{\begin{array}{l}
\left(\left(x^{2}-1\right) u\right)^{\prime}+2 x\left(2 \lambda x^{4}-(2 \lambda+\alpha) x^{2}+\alpha-\beta-1\right) u=0, \\
|\beta|+\left|4 \lambda(u)_{4}-2 \alpha(u)_{2}-2 \beta-1\right| \neq 0 .
\end{array}\right.
$$

This is the irreducible Pearson equation for a linear functional of class four, when $\Phi$ in (10) has two simple zeros.

$\mathbf{B}_{5.2} \cdot \tilde{\Phi}$ has one zero $\alpha_{1}=0$.

We choose $a$ in such a way that $\tilde{a}_{4} a^{4}=4$. Then (41) reads as

$$
\left(x^{2} u\right)^{\prime}+x\left(4 x^{4}+a_{2} x^{2}+a_{0}\right) u=0 .
$$

By a suitable choice of the coefficients $a_{2}$ and $a_{0}$, (44) can be written as

$$
\left\{\begin{array}{l}
\left(x^{2} u\right)^{\prime}+x\left(4 x^{4}-2 \beta x^{2}-\alpha-2\right) u=0 \\
4(u)_{4}-2 \beta(u)_{2}-\alpha-1 \neq 0
\end{array}\right.
$$


This is the irreducible Pearson equation for a linear functional of class four, when $\Phi$ in (10) has a zero of multiplicity two.

$\mathbf{B}_{6} . \tilde{\Phi}$ is a constant.

After the displacement, we get

$$
u^{\prime}+x\left(a^{6} \tilde{a}_{4} x^{4}+a^{4} \tilde{a}_{2} x^{2}+a^{2} \tilde{a}_{0}\right) u=0 .
$$

If we choose $a$ such that $a^{6} \tilde{a}_{4}=6$, then (46) reduces to

$$
u^{\prime}+2 x\left(3 x^{4}+2 \alpha x^{2}+\beta\right) u=0 .
$$

Since $\Phi$ is non zero constant, condition (8) is satisfied and (47) is the irreducible Pearson equation for a linear functional of class four, when $\Phi$ in (10) is a constant.

\section{Integral representation of symmetric semi-classical linear functionals of class $\mathrm{s}=4$.}

Let $u$ be a symmetric semi-classical linear functional of class $s$ satisfying $(10)$ and let us assume $(u)_{0}=1$.

Our aim will be to obtain an integral representation of $u$

$$
\langle u, f(x)\rangle=\int_{-\infty}^{+\infty} f(x) U(x) d x
$$

where we assume the function $U$ is absolutely continuous on $\mathbb{R}$ and it decays as fast as its derivative $U^{\prime}$. From (10), we get

$$
\left.\int_{-\infty}^{+\infty}\left((\Phi U)^{\prime}+\Psi U\right) f(x) d x-\Phi(x) U(x) f(x)\right]_{-\infty}^{+\infty}=0, \quad f \in \mathcal{P}
$$

Hence, from the assumptions on $U$, the following conditions hold

$$
\begin{gathered}
\Phi(x) U(x) f(x)]_{-\infty}^{+\infty}=0, \quad f \in \mathcal{P}, \\
\int_{-\infty}^{+\infty}\left((\Phi U)^{\prime}+\Psi U\right) f(x) d x=0, \quad f \in \mathcal{P} .
\end{gathered}
$$

Condition (50) implies

$$
(\Phi U)^{\prime}+\Psi U=w g
$$

where $w$ is arbitrary and $g$ is a locally integrable function with rapid decay representing the null-linear functional (see [13])

$$
\int_{-\infty}^{+\infty} x^{n} g(x)=0, \quad n \geq 0
$$

Conversely, if $U$ is a solution of (51) verifying the hypothesis above as well as

$$
\int_{-\infty}^{+\infty} U(x) d x \neq 0,
$$

then (49) - (50) are fulfilled and (48) defines a linear functional $u$ which is a solution of (10).

If $w=0$, then (51) becomes

$$
\frac{U^{\prime}}{U}=-\frac{\Phi^{\prime}+\Psi}{\Phi} .
$$

We will consider the above fifteen canonical functional equations and, in each case, an integral representation of the corresponding linear functionals will be given.

\section{Case A.}


$\mathbf{A}_{1}$. From (15) to (54), we get

$$
\frac{U^{\prime}(x)}{U(x)}=\frac{2 \alpha x}{x^{2}-1}+\frac{2 \beta x}{x^{2}-c^{2}}+\frac{2 \gamma x}{x^{2}-d^{2}}
$$

and, as a consequence,

$$
U(x)=\left|x^{2}-1\right|^{\alpha}\left|x^{2}-c^{2}\right|^{\beta}\left|x^{2}-d^{2}\right|^{\gamma}
$$

is the solution at some intervals depending on $c$ and $d$ (see below).

On the other hand, if $\alpha \beta \gamma \neq 0$ and $\alpha, \beta, \gamma>-1$, then the conditions (8) and (49) hold in the following situations:

$\mathbf{s}_{1}: 0<c<d<1$. In such a case, $u$ is represented by

$$
\langle u, f\rangle=\int_{-1}^{1} f(x)\left(1-x^{2}\right)^{\alpha}\left|x^{2}-c^{2}\right|^{\beta}\left|x^{2}-d^{2}\right|^{\gamma}\left(A \chi_{[-c, c]}(x)+B \chi_{[-d, d]}(x)+C \chi_{[-1,1]}(x)\right) d x,
$$

since from an integration by parts we deduce that the linear functionals $u_{1}, u_{2}$ and $u_{3}$ defined by

$$
\left\langle u_{1}, f\right\rangle=\int_{-c}^{c} U(x) f(x) d x,\left\langle u_{2}, f\right\rangle=\int_{-d}^{d} U(x) f(x) d x \text { and }\left\langle u_{3}, f\right\rangle=\int_{-1}^{1} U(x) f(x) d x,
$$

are solutions of (15). The same result holds for any linear combination of $u_{1}, u_{2}$ and $u_{3}$ with coefficients $A, B$ and $C$

$$
\left\langle A u_{1}+B u_{2}+C u_{3}, f\right\rangle=\int_{-1}^{1} U(x)\left(A \chi_{[-c, c]}+B \chi_{[-d, d]}+C \chi_{[-1,1]}\right) f(x) d x
$$

where $\chi_{[a, b]}$ denotes the characteristic function of the interval $[a, b]$, i. e. $\chi_{[a, b]}(x)=1$ when $x \in[a, b]$ and zero otherwise. $A, B$ and $C$ will be chosen in such a way that $(u)_{0}=1$.

We will proceed in a similar way in the case below.

$\mathbf{s}_{2}: d>c>1$. In such a case, we get

$$
\langle u, f\rangle=\int_{-d}^{d} f(x)\left|1-x^{2}\right|^{\alpha}\left|x^{2}-c^{2}\right|^{\beta}\left(d^{2}-x^{2}\right)^{\gamma}\left(A_{1} \chi_{[-c, c]}(x)+B_{1} \chi_{[-d, d]}(x)+C_{1} \chi_{[-1,1]}(x)\right) d x .
$$

Remark 3. The condition $\alpha \beta \gamma \neq 0$ is sufficient to ensure that the condition of irreducibility (8) is satisfied (see second equation in (15)). Indeed, for every parameter $\alpha, \beta, \gamma>-1$ satisfying (15), we obtain the integral representations given above.

\section{Particular case:}

If $\alpha=\beta=\gamma=0$, then (15) becomes

$$
\left\{\begin{array}{l}
\left(\left(x^{2}-1\right)\left(x^{2}-c^{2}\right)\left(x^{2}-d^{2}\right) u\right)^{\prime}+2 x\left(-3 x^{4}+2\left(1++c^{2}+d^{2}\right) x^{2}\right. \\
\left.-c^{2} d^{2}-c^{2}-d^{2}\right) u=0 \\
\left\{-5(u)_{4}+3\left(1+d^{2}\right)(u)_{2}-d^{2}\right\} \times\left\{-5(u)_{4}+3\left(c^{2}+d^{2}\right)(u)_{2}-c^{2} d^{2}\right\} \\
\times\left\{-5(u)_{4}+3\left(1+c^{2}\right)(u)_{2}-c^{2}\right\} \neq 0 .
\end{array}\right.
$$

Which may be written as

$$
\begin{gathered}
\left(x^{2}-1\right)\left(x^{2}-c^{2}\right)\left(x^{2}-d^{2}\right) u^{\prime}=0, \\
\left\{-5(u)_{4}+3\left(1+d^{2}\right)(u)_{2}-d^{2}\right\} \times\left\{-5(u)_{4}+3\left(c^{2}+d^{2}\right)(u)_{2}-c^{2} d^{2}\right\} \times\left\{-5(u)_{4}+3\left(1+c^{2}\right)-c^{2}\right\} \neq 0 .
\end{gathered}
$$

(59) is equivalent to

$$
u^{\prime}=\lambda_{1}\left(\delta_{1}-\delta_{-1}\right)+\lambda_{2}\left(\delta_{c}-\delta_{-c}\right)+\lambda_{3}\left(\delta_{d}-\delta_{-d}\right),
$$

with

$$
\left\{\begin{aligned}
\lambda_{1} & =\frac{3\left(c^{2}+d^{2}\right)(u)_{2}-5(u)_{4}-c^{2} d^{2}}{2\left(c^{2}-1\right)\left(d^{2}-1\right)} \\
\lambda_{2} & =\frac{3\left(1+d^{2}\right)(u)_{2}-5(u)_{4}-d^{2}}{2 c\left(c^{2}-1\right)\left(c^{2}-d^{2}\right)} \\
\lambda_{3} & =\frac{3\left(c^{2}+1\right)(u)_{2}-5(u)_{4}-c^{2}}{2 d\left(d^{2}-1\right)\left(d^{2}-c^{2}\right)}
\end{aligned}\right.
$$


(60) is equivalent to $\lambda_{1} \lambda_{2} \lambda_{3} \neq 0$.

On the one hand, by virtue of the definition of the derivative of a linear functional, we have

$$
\left(u^{\prime}\right)_{2 n+1}=-(2 n+1)(u)_{2 n}, n \geq 0 .
$$

On the other hand, from (61) we get

$$
\left(u^{\prime}\right)_{2 n+1}=2\left(\lambda_{1}+\lambda_{2} c^{2 n+1}+\lambda_{3} d^{2 n+1}\right), n \geq 0 .
$$

The conjunction of (63)) and (64) leads to

$$
(u)_{2 n}=-\frac{2\left(\lambda_{1}+\lambda_{2} c^{2 n+1}+\lambda_{3} d^{2 n+1}\right)}{2 n+1}, n \geq 0 .
$$

By a simple computation, we can see that $(u)_{0}=1$ and $(u)_{2},(u)_{4}$ are an arbitrary parameter.

Let us define

$E_{0}=\left\{(\lambda, \mu) \in \mathbb{C}^{2} / \lambda_{1} \lambda_{2} \lambda_{3}=0\right\}$,

$E_{n}=\left\{(\lambda, \mu) \in \mathbb{C}^{2} / \Delta_{n}(\lambda, \mu)=0\right\}, n \geq 1$,

where $\lambda=(u)_{2}, \mu=(u)_{4}$ and $\Delta_{n}(\lambda, \mu)$ is the Hankel determinant associated with $u$.

For any integer $n, \Delta_{n}(\lambda, \mu)$ is a polynomial in $\lambda$ and $\mu$, then the linear functional $u$ satisfying (58) is regular and of class four if and only if $(\lambda, \mu) \notin \bigcup_{n \geq 0} E_{n}$.

Let us back to equation (61), then we have

$$
u=\lambda_{1}\left(H_{1}-H_{-1}\right)+\lambda_{2}\left(H_{c}-H_{-c}\right)+\lambda_{3}\left(H_{d}-H_{-d}\right),
$$

where $H_{x}$ is the Heaviside step function:

$$
H_{x}= \begin{cases}1 & \text { over }] x,+\infty[ \\ 0 & \text { over }]-\infty, x]\end{cases}
$$

Then, $u$ is represented by

$$
\langle u, f\rangle=-\lambda_{1} \int_{-1}^{1} f(x) d x-\lambda_{2} \int_{-c}^{c} f(x) d x-\lambda_{3} \int_{-d}^{d} f(x) d x .
$$

$\mathbf{A}_{2}$. From (17) to (54), we have

$$
\frac{U^{\prime}(x)}{U(x)}=\frac{\alpha}{x}+\frac{2 \beta x}{x^{2}-1}+\frac{2 \gamma x}{x^{2}-c^{2}},
$$

so

$$
U(x)=|x|^{\alpha}\left|1-x^{2}\right|^{\beta}\left|x^{2}-c^{2}\right|^{\gamma} .
$$

Finally, it is straightforward to check that for $\beta \gamma \neq 0$ and $\alpha, \beta, \gamma>-1$, the conditions (8) and (49) hold in the following situations:

$\mathbf{s}_{1}: 0<c<1$. In such a case, $u$ is represented by

$$
\langle u, f\rangle=\int_{-1}^{1} f(x)|x|^{\alpha}\left(1-x^{2}\right)^{\beta}\left|x^{2}-c^{2}\right|^{\gamma}\left(A \chi_{[-c, c]}+B \chi_{[-1,1]}\right) d x
$$

$\mathbf{s}_{2}: c>1$. In such a case, we get

$$
\langle u, f\rangle=\int_{-c}^{c} f(x)|x|^{\alpha}\left|1-x^{2}\right|^{\beta}\left(c^{2}-x^{2}\right)^{\gamma}\left(A_{1} \chi_{[-c, c]}+B_{1} \chi_{[-1,1]}\right) d x .
$$

$\mathbf{A}_{3}$. From (19) to (54), we obtain

$$
\frac{U^{\prime}}{U}=\frac{2 \alpha x}{x^{2}-1}-\frac{2 \beta x}{\left(x^{2}-1\right)^{2}}+\frac{2 \gamma x}{x^{2}-c^{2}},
$$


and, as a consequence,

$$
U(x)=\left|x^{2}-1\right|^{\alpha}\left|x^{2}-c^{2}\right|^{\gamma} e^{\frac{-\beta}{1-x^{2}}},
$$

is the solution at some intervals depending on $c$. For instance, if $\beta \gamma \neq 0, \alpha, \gamma>-1$ and $\beta>0$, then the conditions (8) and (49) hold in the following situations:

$\mathbf{s}_{1}$ : If $\left.c \in\right] 0,1[$, then

$$
\langle u, f\rangle=\int_{-1}^{1} f(x)\left(1-x^{2}\right)^{\alpha}\left|x^{2}-c^{2}\right|^{\gamma} e^{\frac{-\beta}{1-x^{2}}}\left(A \chi_{[-c, c]}+B \chi_{[-1,1]}\right) d x .
$$

$\mathbf{s}_{2}$ : If $c>1$, then $u$ is represented by

$$
\langle u, f\rangle=\int_{-c}^{c} f(x)\left|1-x^{2}\right|^{\alpha}\left(c^{2}-x^{2}\right)^{\gamma} e^{\frac{-\beta}{1-x^{2}}}\left(A_{1} \chi_{[-c, c]}+B_{1} \chi_{[-1,1]}\right) d x .
$$

Remark 4. For every parameter $\alpha, \gamma>-1$ and $\beta>0$ such that (19) is verified, we also obtain the integral representation given above, except when $\beta \leq 0$. For $\beta=0$ see below.

Particular case:

If $\beta=0$, thus for (19) we get

$$
\left\{\begin{array}{l}
\left(\left(x^{2}-1\right)^{2}\left(x^{2}-c^{2}\right) u\right)^{\prime}+2 x\left(x^{2}-1\right)\left(-(\alpha+\gamma+3) x^{2}+(\alpha+2) c^{2}+\gamma+1\right) u=0 \\
\left\{-(2 \alpha+2 \gamma+5)(u)_{4}+\left((2 \alpha+3) c^{2}+2 \gamma+3\right)(u)_{2}-c^{2}\right\} \\
\times\left\{|\gamma|+\left|-(2 \alpha+2 \gamma+5)(u)_{4}+2\left(3+\alpha+\gamma\left(2-c^{2}\right)\right)(u)_{2}+2 \gamma c^{2}\left(1-c^{2}\right)-2 \gamma-1\right|\right\} \neq 0 .
\end{array}\right.
$$

Then,

$$
\left(x^{2}-1\right) u=k \hat{v},
$$

where $k$ is a normalization term and $\hat{v}$ is the symmetric semi-classical linear form of class two defined by the Pearson equation ( see $[8,10]$ )

$$
\left\{\begin{array}{l}
\left(\left(x^{2}-1\right)\left(x^{2}-c^{2}\right) \hat{v}\right)^{\prime}+2 x\left(-(\alpha+\gamma+3) x^{2}+(\alpha+2) c^{2}+\gamma+1\right) \hat{v}=0 \\
\left\{|\alpha|+\left|-(2 \alpha+2 \gamma+3)(\hat{v})_{2}+(2 \alpha+1) c^{2}\right|\right\} \\
\times\left\{|\gamma|+\left|-(2 \alpha+2 \gamma+3)(\hat{v})_{2}+2 \gamma\left(1-c^{2}\right)+1\right|\right\} \neq 0 .
\end{array}\right.
$$

Notice that the linear functional $u$ defined by (72) is regular if and only if the MOPS $P_{n}(x), n \geq 0$, satisfies (see [6])

$$
\left|\begin{array}{cc}
P_{n+1}(-1 ; k) & P_{n}(-1 ; k) \\
P_{n+1}(1 ;-k) & P_{n}(1 ;-k)
\end{array}\right| \neq 0, n \geq 0,
$$

where $P_{n}(x), n \geq 0$, are the monic polynomials orthogonal with respect to $\hat{v}$ and $\left\{P_{n}(. ; \mu)\right\}_{n \geq 0}$ is the co-recursive MOPS of $\left\{P_{n}\right\}_{n \geq 0}$ (see [7])

$$
P_{n}(x ; \mu)=P_{n}(x)-\mu P_{n-1}^{(1)}(x),
$$

where $\left\{P_{n}^{(1)}\right\}_{n \geq 0}$ is the sequence of associated polynomials of the first kind for the sequence $\left\{P_{n}\right\}_{n \geq 0}$ (see [15]). From (3) and (72), we obtain

$$
u=k\left(x^{2}-1\right)^{-1} \hat{v}+\frac{1}{2}\left(\delta_{1}+\delta_{-1}\right) .
$$

$\mathbf{A}_{4}$. From (21) to (54), we get

$$
\frac{U^{\prime}}{U}=\frac{2 \beta x}{x^{2}-1}-\frac{2 \gamma x}{\left(x^{2}-1\right)^{2}}+\frac{2 \alpha+1}{x},
$$


therefore,

$$
U(x)=|x|^{2 \alpha+1}\left|x^{2}-1\right|^{\beta} e^{\frac{-\gamma}{1-x^{2}}} .
$$

Notice that if $\gamma>0$ and $\alpha, \beta>-1$, then the conditions (8) and (49) hold.

Thus $u$ is represented by

$$
\langle u, f\rangle=k \int_{-1}^{1} f(x)|x|^{2 \alpha+1}\left(1-x^{2}\right)^{\beta} e^{\frac{-\gamma}{1-x^{2}}} d x .
$$

Here $k$ is a constant such that $(u)_{0}=1$.

\section{Particular case:}

If $\gamma=0$, thus from (21) we have

$$
\left\{\begin{array}{l}
\left(x^{2}\left(x^{2}-1\right)^{2} u\right)^{\prime}+x\left(-(2 \alpha+2 \beta+7) x^{4}+2(2 \alpha+\beta+5) x^{2}-2 \alpha-3\right) u=0 \\
\left\{-(\alpha+\beta+3)(u)_{4}+(2 \alpha+\beta+4)(u)_{2}-\alpha-1\right\} \\
\times\left\{-(\alpha+\beta+3)(u)_{4}+(\alpha+2)(u)_{2}\right\} \neq 0
\end{array}\right.
$$

Hence

$$
u=\left(1-k_{1}-2 k_{2}\right) \mathcal{G} \cdot \mathcal{G}(\beta, \alpha)+k_{1} \delta_{0}+k_{2}\left(\delta_{1}+\delta_{-1}\right),
$$

where $\mathcal{G} \cdot \mathcal{G}(a, b)$ is the generalized Gegenbauer linear functional defined by the Pearson equation (see [7])

$$
\left(x\left(x^{2}-1\right) \mathcal{G} \cdot \mathcal{G}(a, b)\right)^{\prime}+2\left(-(a+b+2) x^{2}+b+1\right) \mathcal{G} \cdot \mathcal{G}(a, b) .
$$

The above linear functional $u$ is regular if and only if $k_{1}$ and $k_{2}$ satisfies (see [2])

$$
\left|\begin{array}{ccc}
1+k_{1} K_{n}(0,0) & k_{2} K_{n}(0,1) & k_{2} K_{n}(0,-1) \\
k_{1} K_{n}(1,0) & 1+k_{2} K_{n}(1,1) & k_{2} K_{n}(1,-1) \\
k_{1} K_{n}(-1,0) & k_{2} K_{n}(-1,1) & 1+k_{2} K_{n}(-1,-1)
\end{array}\right| \neq 0, n \geq 0
$$

where $K_{n}(x, y):=\sum_{m=0}^{n} \frac{P_{m}^{(\beta, \alpha)}(x) P_{m}^{(\beta, \alpha)}(y)}{\left\langle\mathcal{G G}(\beta, \alpha),\left(P_{m}^{(\beta, \alpha)}\right)^{2}\right\rangle}$ is the $n$-th reproducing Kernel associated with the linear functional $\mathcal{G G}(\beta, \alpha)$ and $P_{n}^{(\beta, \alpha)}(x), n \geq 0$, are the monic polynomials orthogonal with respect to $\mathcal{G G}(\beta, \alpha)$.

The action of the linear functionals defined in (76) over the polynomial $x^{2 n}$ yields

$$
(u)_{2 n}=\left(1-k_{1}-2 k_{2}\right) \frac{\Gamma(n+\alpha+1) \Gamma(\alpha+\beta+2)}{\Gamma(n+\alpha+\beta+2) \Gamma(\alpha+1)}+2 k_{2}, \quad n \geq 1 .
$$

Finally from (76) we obtain

$$
\langle u, f\rangle=\left(1-k_{1}-2 k_{2}\right) \frac{\Gamma(\alpha+\beta+2)}{\Gamma(\alpha+1) \Gamma(\beta+1)} \int_{-1}^{1}|x|^{2 \alpha+1}\left(1-x^{2}\right)^{\beta} f(x) d x+k_{1} f(0)+k_{2}(f(1)+f(-1)) .
$$

$\mathbf{A}_{5}$. From (23) to (54), we have

$$
\frac{U^{\prime}}{U}=\frac{2 \gamma x}{x^{2}-1}+\frac{2 \beta}{x^{3}}+\frac{\alpha}{x}
$$

Thus,

$$
U(x)=|x|^{\alpha}\left|x^{2}-1\right|^{\gamma} e^{\frac{-\beta}{x^{2}}} .
$$

Notice that if $\gamma \neq 0, \alpha, \gamma>-1$ and $\beta \geq 0$, then the conditions (8) and (49) hold.

Thus $u$ is represented by

$$
\langle u, f\rangle=k \int_{-1}^{1} f(x)|x|^{\alpha}\left(1-x^{2}\right)^{\gamma} e^{\frac{-\beta}{x^{2}}} d x .
$$

$\mathbf{A}_{6}$. From (25) to (54) we obtain

$$
\frac{U^{\prime}}{U}=\frac{2 \alpha x}{x^{2}-1}+\frac{4 \beta x}{\left(x^{2}-1\right)^{3}},
$$


hence,

$$
U(x)=\left|x^{2}-1\right|^{\alpha} e^{\frac{-\beta}{\left(x^{2}-1\right)^{2}}} .
$$

Furthermore, for $\beta>0$ and $\alpha>-1$, (8) and (49) hold.

As a consequence $u$ is represented by

$$
\langle u, f\rangle=A \int_{-1}^{1} f(x)\left(1-x^{2}\right)^{\alpha} e^{\frac{-\beta}{\left(x^{2}-1\right)^{2}}} d x .
$$

Particular case:

If $\beta=0$, then (25) becomes

$$
\left\{\begin{array}{l}
\left(\left(x^{2}-1\right)^{3} u\right)^{\prime}-2(\alpha+3) x\left(x^{2}-1\right)^{2} u=0 \\
-(2 \alpha+5)(u)_{4}+2(\alpha+3)(u)_{2}-1 \neq 0
\end{array}\right.
$$

Thus,

$$
u=\mathcal{G}(\alpha)+k\left(\delta_{1}^{\prime}-\delta_{-1}^{\prime}\right),
$$

where $\mathcal{G}(\alpha)$ is the Gegenbauer linear functional that satisfies (see [15])

$$
\left(\left(x^{2}-1\right) \mathcal{G}(\alpha)\right)^{\prime}-2(\alpha+1) x \mathcal{G}(\alpha)=0,
$$

with $\alpha+1 \neq-n$ and $2 \alpha+1 \neq-n, n \geq 0$.

The above linear functional $u$ is regular if and only if $k$ satisfies (see [2])

$$
\left|\begin{array}{cc}
1-k K_{n}(1,1) & k K_{n}(1,-1) \\
-k K_{n}(-1,1) & 1+k K_{n}(-1,-1)
\end{array}\right| \neq 0, n \geq 0 .
$$

Finally, from (81) we get

$$
\langle u, f\rangle=\frac{\Gamma\left(\alpha+\frac{3}{2}\right)}{\sqrt{\pi} \Gamma(\alpha+1)} \int_{-1}^{1}\left(1-x^{2}\right)^{\alpha} f(x) d x-k\left(f^{\prime}(1)-f^{\prime}(-1)\right) .
$$

When $f(x)=x^{2 n}$, the above expression becomes

$$
(u)_{2 n}=\Gamma\left(n+\frac{1}{2}\right) \frac{\Gamma\left(\alpha+\frac{3}{2}\right)}{\sqrt{\pi} \Gamma\left(n+\alpha+\frac{3}{2}\right)}-4 n k, \quad n \geq 0 .
$$

$\mathbf{A}_{7.1}$. In this case, it is not possible to choose $w=0$. Indeed, from (28) and (54) (e.i. (51) with $w=0$ ), we get

$$
\frac{U^{\prime}}{U}=\frac{2 \alpha-5}{x}+\frac{2 \beta}{x^{3}}+\frac{8}{x^{5}}
$$

A priori there is no a real path $\mathcal{C}$ such that $\left.x^{6} U(x) f(x)\right|_{\mathcal{C}}=0, f \in \mathcal{P}$. This is analog of Bessel in classical case (see [13] for more information ).

Thus, we handle it differently with choice $w \neq 0$ in (51).

From (28) to (51), we get

$$
\left(x^{6} U\right)^{\prime}+x\left\{-(2 \alpha+1) x^{4}-2 \beta x^{2}-8\right\} U=w g(x) .
$$

For instance, let $g(x)=x|x| \mathrm{s}\left(x^{2}\right), x \in \mathbb{R}$, where $\mathrm{s}$ is the classical Stieltjes function [13]

$$
\mathrm{s}(x)= \begin{cases}0, & x \leq 0, \\ e^{-x^{\frac{1}{4}}} \sin x^{\frac{1}{4}}, & x>0 .\end{cases}
$$

A possible solution of (82) is the even function

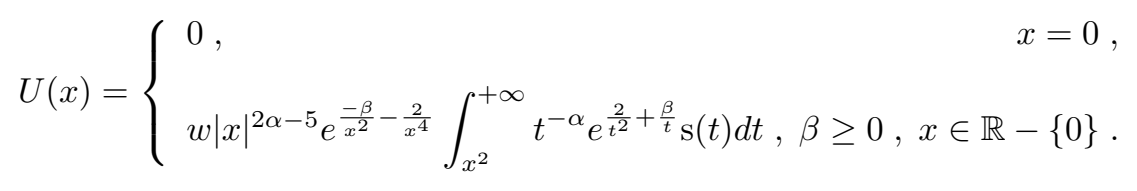


First, condition (49) is fulfilled since

$$
\left|x^{6} U(x)\right| \leq|w||x|^{2 \alpha+1} e^{\frac{-\beta}{x^{2}}-\frac{2}{x^{4}}} \int_{x^{2}}^{+\infty} t^{-\alpha} e^{\frac{2}{t^{2}}+\frac{\beta}{t}} e^{-t^{\frac{1}{4}}} d t=o\left(e^{-\frac{1}{2}|x|^{\frac{1}{2}}}\right), \quad|x| \rightarrow+\infty .
$$

Furthermore, when $x \rightarrow+\infty$

$$
|U(x)| \leq|w| x^{2 \alpha-5} \int_{x^{2}}^{+\infty} t^{-\alpha} e^{-t^{\frac{1}{4}}} d t=o\left(e^{-\frac{1}{2} x^{\frac{1}{2}}}\right)
$$

and when $x \rightarrow+0$

$$
|U(x)| \leq|w| x^{2 \alpha-5} e^{\frac{-\beta}{x^{2}}-\frac{2}{x^{4}}} \int_{x^{2}}^{1} t^{-\alpha} e^{\frac{2}{t^{2}}+\frac{\beta}{t}} d t+o(1) .
$$

Applying l'Hospital's rule to the ratio

$$
\lim _{x \rightarrow+0} \frac{\int_{x^{2}}^{1} t^{-\alpha} e^{\frac{2}{t^{2}}+\frac{\beta}{t}} d t}{x^{-2 \alpha+5} e^{\frac{2}{x^{2}}+\frac{\beta}{x}}}=\lim _{x \rightarrow+0} \frac{2 x}{(2 \alpha-5) x^{4}+2 \beta x^{2}+8}=0,
$$

we get $\lim _{x \rightarrow+0} U(x)=0=U(0)$.

Consequently, $U \in L_{1}$.

Condition (53) now becomes

$$
\int_{-\infty}^{+\infty} U(x) d x=2 w \int_{0}^{+\infty} \xi^{-\alpha} e^{\frac{2}{\xi^{2}}+\frac{\beta}{\xi}} \mathrm{s}(\xi)\left(\int_{0}^{\sqrt{\xi}} x^{2 \alpha-5} e^{\frac{-\beta}{x^{2}}-\frac{2}{x^{4}}} d x\right) d \xi=w S_{\alpha},
$$

with

$$
\begin{gathered}
S_{\alpha}=8 \int_{0}^{+\infty} t^{3-4 \alpha} e^{\frac{2}{t^{8}}+\frac{\beta}{t^{4}}} e^{-t} \varphi_{\alpha-1}\left(t^{2}\right) \sin t d t, \\
\varphi_{\alpha}(t)=\int_{0}^{t} x^{2 \alpha-3} e^{\frac{-\beta}{x^{2}}-\frac{2}{x^{4}}} d x .
\end{gathered}
$$

Let us establish some results about $S_{\alpha}$.

Lemмa 3.1. We have for $\alpha \geq \frac{3}{2}$ and $\beta \geq 0$

$$
\begin{gathered}
\frac{1}{2} t^{2} \varphi_{\alpha}(t) \leq \varphi_{\alpha+1}(t) \leq t^{2} \varphi_{\alpha}(t), \quad t \geq 0 \\
\frac{t^{2 \alpha+2} e^{-\frac{\beta}{t^{2}}-\frac{2}{t^{4}}}}{2(\alpha+1) t^{4}+2 \beta t^{2}+8} \leq \varphi_{\alpha}(t) \leq 2 \frac{t^{2 \alpha+2} e^{-\frac{\beta}{t^{2}}-\frac{2}{t^{4}}}}{(\alpha+1) t^{4}+2 \beta t^{2}+16}, \quad t \geq 0 .
\end{gathered}
$$

Proof. It is easy to prove (88) from (87) and monotonicity.

From (87), we have upon integration by parts

$$
\varphi_{\alpha}(t)=\frac{1}{8} t^{2 \alpha+2} e^{-\frac{\beta}{t^{2}}-\frac{2}{t^{4}}}-\frac{\alpha+1}{4} \varphi_{\alpha+2}(t)-\frac{\beta}{4} \varphi_{\alpha+1}(t), t \geq 0 .
$$

Now, in accordance of (88) and (90) we obtain the desired result (89).

Proposition 3.2. We have the following expression for $n \geq 1$ and $\alpha \in \mathbb{C}$

$$
\begin{aligned}
S_{\alpha} & =\frac{(-1)^{n}}{2^{2 n-3}} \prod_{k=0}^{n-1}(\alpha+2 k) \int_{0}^{+\infty} t^{3-4 \alpha} e^{\frac{\beta}{t^{2}}+\frac{2}{t^{4}}} e^{-t} \varphi_{\alpha+2 n-1}\left(t^{2}\right) \sin t d t \\
& +\beta \sum_{k=0}^{n-1} \frac{(-1)^{k+1}}{2^{2 k-1}} \prod_{i=0}^{k-1}(\alpha+2 i) \int_{0}^{+\infty} t^{3-4 \alpha} e^{\frac{\beta}{t^{2}}+\frac{2}{t^{4}}} e^{-t} \varphi_{\alpha+2 k}\left(t^{2}\right) \sin t d t
\end{aligned}
$$

with $\prod_{0}^{-1}=1$. 
Proof. From (90) and using the Stieltjes representation (52) of the null-form, we get

$$
S_{\alpha}=-2 \alpha \int_{0}^{+\infty} t^{3-4 \alpha} e^{\frac{\beta}{t^{2}}+\frac{2}{t^{4}}} e^{-t} \varphi_{\alpha+1}\left(t^{2}\right) \sin t d t-2 \beta \int_{0}^{+\infty} t^{3-4 \alpha} e^{\frac{\beta}{t^{2}}+\frac{2}{t^{4}}} e^{-t} \varphi_{\alpha}\left(t^{2}\right) \sin t d t .
$$

Suppose (91) for $n \geq 1$ fixed. From (90) where $\alpha \rightarrow \alpha+2 n$ and $t \rightarrow t^{2}$

$$
\varphi_{\alpha+2 n-1}\left(t^{2}\right)=\frac{1}{8} t^{4 \alpha+8 n} e^{-\frac{\beta}{t^{4}}-\frac{2}{t^{8}}}-\frac{\alpha+2 n}{4} \varphi_{\alpha+2 n+1}\left(t^{2}\right)-\frac{\beta}{4} \varphi_{\alpha+2 n}\left(t^{2}\right),
$$

hence easily (91) for $n \rightarrow n+1$.

Corollary 3.3. If $\beta=0$, then $S_{-2 n}=0, n \geq 0$.

This results is consistent with the fact the linear form $u$ defined by (28) where $\beta=0$ is not regular for these values of $\alpha$.

Proposition 3.4. For $\alpha \geq \frac{5}{2}$, we have $S_{\alpha}>0$.

Proof. First, we need the following Lemma [13]

Lemma 3.5. Consider the following integral

$$
S=\int_{0}^{+\infty} F(t) \sin t d t
$$

where we suppose $F(t) \geq 0$, continuous, increasing in $0<t \leq \bar{t}$ and decreasing to zero for $t>\bar{t}$.

Then,

$$
0<\bar{t} \leq \pi, \quad \int_{0}^{\pi}(F(t)-F(t+\pi)) \sin t d t \leq 0 \Rightarrow S>0 .
$$

Now, denoting $F(t)=F_{\alpha}(t)=f_{\alpha}(t) e^{-t}$ with

$$
f_{\alpha}(t)=t^{3-4 \alpha} e^{\frac{\beta}{t^{2}}+\frac{2}{t^{4}}} \varphi_{\alpha-1}\left(t^{2}\right) .
$$

We have from (89)

$$
\frac{t^{3}}{2 \alpha t^{8}+2 \beta t^{4}+8} \leq f_{\alpha}(t) \leq \frac{2 t^{3}}{\alpha t^{8}+2 \beta t^{4}+16}, \quad t \geq 0, \quad \alpha \geq \frac{5}{2} .
$$

Then,

$$
\frac{t^{3} e^{-t}}{2 \alpha t^{8}+2 \beta t^{4}+8} \leq F_{\alpha}(t) \leq 2 \frac{t^{3} e^{-t}}{\alpha t^{8}+2 \beta t^{4}+16}, \quad t \geq 0, \quad \alpha \geq \frac{5}{2} .
$$

Consequently, $F_{\alpha}(t)>0$ for $t>0, F_{\alpha}(0)=0$ and $\lim _{t \rightarrow+\infty} F_{\alpha}(t)=0$ which implies that $F_{\alpha}$ has a maximum for $t=\bar{t}$ defined by $f_{\alpha}^{\prime}(\bar{t})=f_{\alpha}(\bar{t})$.

Hence

$$
f_{\alpha}(\bar{t})=\frac{2 \bar{t}^{3}}{\bar{t}^{9}+(4 \alpha-3) \bar{t}^{8}+4 \beta \bar{t}^{4}+16},
$$

since

$$
f_{\alpha}^{\prime}(t)=\frac{2}{t^{6}}-\left\{\frac{4 \alpha-3}{t}+\frac{4 \beta}{t^{5}}+\frac{16}{t^{9}}\right\} f_{\alpha}(t), t>0 .
$$

From the first inequality of (94) and by virtue of (96) necessarily $\bar{t} \leq 3$.

Therefore the implication (93) is true if the following is verified

$$
\int_{0}^{\pi} \sin t \frac{2(\pi+t)^{3}}{\alpha(\pi+t)^{8}+2 \beta(\pi+t)^{4}+16} e^{-t-\pi} d t \leq \int_{0}^{\pi} \sin t \frac{t^{3}}{2 \alpha t^{8}+2 \beta t^{4}+8} e^{-t} d t .
$$


The function $t \rightarrow \frac{2 t^{3}}{\alpha t^{8}+2 \beta t^{4}+16}$ is decreasing for $t \geq t_{1}=\left(\frac{\sqrt{240 \alpha+\beta^{2}}-\beta}{5 \alpha}\right)^{\frac{1}{4}}$ and from $\alpha \geq \frac{5}{2}$ we have easily $t_{1} \leq$ $\left(\frac{4}{5} \sqrt{6}\right)^{\frac{1}{4}}<\frac{\pi}{2}$. We have successively

$$
\int_{0}^{\pi} \sin t \frac{2(t+\pi)^{3}}{\alpha(t+\pi)^{8}+2 \beta(t+\pi)^{4}+16} e^{-t-\pi} d t \leq e^{-\pi} \frac{\pi^{3}}{\alpha \pi^{8}+2 \beta \pi^{4}+16}\left(1+e^{-\pi}\right) .
$$

On the other hand

$$
\begin{aligned}
\int_{t_{1}}^{\pi} \sin t \frac{t^{3}}{2 \alpha t^{8}+2 \beta t^{4}+8} e^{-t} d t & =\int_{t_{1}}^{\pi} \sin t \frac{t^{3}}{\alpha t^{8}+2 \beta t^{4}+16} \frac{\alpha t^{8}+2 \beta t^{4}+16}{2 \alpha t^{8}+2 \beta t^{4}+8} e^{-t} d t \\
& \geq \frac{1}{2} \frac{\pi^{3}}{\alpha \pi^{8}+2 \beta \pi^{4}+16} \int_{\frac{\pi}{2}}^{\pi} \sin t e^{-t} d t \\
& \geq \frac{1}{4} e^{-\pi} \frac{\pi^{3}}{\alpha \pi^{8}+2 \beta \pi^{4}+16}\left(1+e^{\frac{\pi}{2}}\right) .
\end{aligned}
$$

Thus, (97) is fulfilled if

$$
e^{-\pi} \frac{\pi^{3}}{\alpha \pi^{8}+2 \beta \pi^{4}+16}\left(1+e^{-\pi}\right) \leq \int_{0}^{t_{1}} \sin t \frac{2 t^{3}}{\alpha t^{8}+2 \beta t^{4}+16} e^{-t} d t+\frac{1}{4} e^{-\pi} \frac{\pi^{3}\left(1+e^{\frac{\pi}{2}}\right)}{\alpha \pi^{8}+2 \beta \pi^{4}+16} .
$$

But, $1+e^{-\pi}<\frac{1}{4}\left(1+e^{\frac{\pi}{2}}\right)$, therefore the inequality (98) is satisfied and the proposition is proved.

Finally, for $f \in \mathcal{P}, \alpha \geq \frac{5}{2}$

$$
\langle u, f\rangle=S_{\alpha}^{-1} \int_{-\infty}^{+\infty} \frac{1}{|x|^{5}} \int_{x^{2}}^{+\infty}\left(\frac{x^{2}}{t}\right)^{\alpha} \exp \left(-\frac{\beta}{x^{2}}+\frac{\beta}{t}\right) \exp \left(\frac{2}{t^{2}}-\frac{2}{x^{4}}\right) s(t) d t f(x) d x .
$$

Remark 5. Multiplying (28) by $x$ and using (1) we get

$$
\left(x^{7} u\right)^{\prime}-2 x^{2}\left((\alpha+1) x^{4}+\beta x^{2}+4\right) u=0 .
$$

Applying the operator $\sigma$ for the above equation and using (4) - (5), we obtain

$$
\left(x^{4} \sigma u\right)^{\prime}-x\left((\alpha+1) x^{2}+\beta x+4\right) \sigma u=0 .
$$

Moreover, since the linear form $u$ is symmetric and regular, then $\sigma u$ is regular.

If $\alpha(u)_{4}+\beta(u)_{2}+4 \neq 0$, then from (8), the linear functional $\sigma u$ is semi-classical of class $s=2$.

From (99), we get

$$
\begin{aligned}
\langle\sigma u, f(x)\rangle & =\left\langle u, f\left(x^{2}\right)\right\rangle \\
& =S_{\alpha}^{-1} \int_{-\infty}^{+\infty} \frac{1}{|x|^{5}} \int_{x^{2}}^{+\infty}\left(\frac{x^{2}}{t}\right)^{\alpha} \exp \left(-\frac{\beta}{x^{2}}+\frac{\beta}{t}\right) \exp \left(\frac{2}{t^{2}}-\frac{2}{x^{4}}\right) s(t) d t f\left(x^{2}\right) d x .
\end{aligned}
$$

After a change of variables, we get

$$
\langle\sigma u, f(x)\rangle=S_{\alpha}^{-1} \int_{0}^{+\infty} x^{\alpha-3} \exp \left(\frac{-\beta}{x}-\frac{2}{x^{2}}\right) \int_{x}^{+\infty} t^{-\alpha} \exp \left(\frac{2}{t^{2}}+\frac{\beta}{t}\right) s(t) d t f(x) d x .
$$

The integral representation (100) doesn't exist in the list given in [10].

$\mathbf{A}_{7.2}$. By applying the same process as we did to obtain (99) with $g(x)=x|x|^{3} \mathrm{~s}\left(x^{2}\right)$, we get after some straightforward calculations

$$
\langle u, f\rangle=T_{\alpha}^{-1} \int_{-\infty}^{+\infty} \frac{1}{|x|^{3}} \int_{x^{2}}^{+\infty}\left(\frac{t^{2}}{x^{4}}\right)^{1-\alpha} \exp \left(\frac{2}{t}-\frac{2}{x^{2}}\right) s(t) d t f(x) d x, \quad \alpha \geq \frac{7}{4},
$$

with

$$
T_{\alpha}=8 \int_{0}^{+\infty} t^{11-8 \alpha} e^{\frac{2}{t^{4}}} e^{-t} \psi_{\alpha-\frac{3}{2}}\left(t^{2}\right) \sin t d t>0
$$




$$
\psi_{\alpha}(t)=\int_{0}^{t} x^{4 \alpha-1} e^{\frac{-2}{x^{2}}} d x
$$

Besides $T_{\alpha}$ and $\psi_{\alpha}$ verify the following relation:

$$
\begin{gathered}
\frac{1}{2} t^{2} \psi_{\alpha}(t) \leq \psi_{\alpha+\frac{1}{2}}(t) \leq t^{2} \psi_{\alpha}(t), \alpha \geq \frac{1}{4}, t \geq 0, \\
\frac{t^{4 \alpha+2} e^{\frac{-2}{t^{2}}}}{4+2(2 \alpha+1) t^{2}} \leq \psi_{\alpha}(t) \leq \frac{t^{4 \alpha+2} e^{\frac{-2}{t^{2}}}}{4+(2 \alpha+1) t^{2}}, \alpha \geq \frac{1}{4}, t \geq 0, \\
T_{\alpha}=\frac{(-1)^{n}}{2^{n-3}} \prod_{k=0}^{n-1}(2 \alpha-2+k) \int_{0}^{+\infty} t^{11-8 \alpha} e^{\frac{2}{t^{4}}} e^{-t} \psi_{\alpha-1+\frac{n-1}{2}}\left(t^{2}\right) \sin t d t, n \geq 1 .
\end{gathered}
$$

Remark 6. By applying the same process as we did to obtain (100), we have

$$
\left(x^{4} \sigma u\right)^{\prime}-2 x^{2}(\alpha x+1) \sigma u=0 .
$$

Moreover, if $(1-2 \alpha)(u)_{4}-2(u)_{2} \neq 0$, then from (8), the linear functional $\sigma u$ is semi-classical of class $s=2$. Finally, from (102), we get

$$
\langle\sigma u, f(x)\rangle=T_{\alpha}^{-1} \int_{0}^{+\infty} x^{2 \alpha-4} e^{-\frac{2}{x}} \int_{x}^{+\infty} t^{2-2 \alpha} e^{\frac{2}{t}} s(t) d t f(x) d x .
$$

The integral representation (108) doesn't exist in the list given in [10].

\section{Case B.}

$\mathbf{B}_{1}$. From (34) to (54), we have

$$
\frac{U^{\prime}(x)}{U(x)}=-2 \lambda x+\frac{2 \alpha x}{x^{2}-1}+\frac{2 \beta x}{x^{2}-c^{2}}
$$

Thus,

$$
U(x)=\left|1-x^{2}\right|^{\alpha}\left|x^{2}-c^{2}\right|^{\beta} e^{-\lambda x^{2}},
$$

is the solution in $]-\infty,+\infty[,[-1,1]$ and $[-c, c]$.

Furthermore, for $\alpha \beta \neq 0$ and $\alpha, \beta>-1,(8)$ and (49) hold in the following situations:

$s_{1}:$ If $\lambda>0$, then $u$ is represented by

$$
\langle u, f\rangle=\int_{-\infty}^{+\infty}\left|1-x^{2}\right|^{\alpha}\left|x^{2}-c^{2}\right|^{\beta} e^{-\lambda x^{2}}\left(A \chi_{[-1,1]}(x)+B \chi_{[-c, c]}(x)+C \chi_{]-\infty,+\infty}(x)\right) f(x) d x .
$$

$s_{2}:$ If $\lambda<0$ and $0<c<1$, then

$$
\langle u, f\rangle=\int_{-1}^{1}\left(1-x^{2}\right)^{\alpha}\left|x^{2}-c^{2}\right|^{\beta} e^{-\lambda x^{2}}\left(A_{1} \chi_{[-1,1]}(x)+B_{1} \chi_{[-c, c]}(x)\right) f(x) d x .
$$

$s_{3}:$ If $\lambda<0$ and $c>1$, then

$$
\langle u, f\rangle=\int_{-c}^{c}\left|1-x^{2}\right|^{\alpha}\left(c^{2}-x^{2}\right)^{\beta} e^{-\lambda x^{2}}\left(A_{2} \chi_{[-1,1]}(x)+B_{2} \chi_{[-c, c]}(x)\right) f(x) d x .
$$

$\mathbf{B}_{2}$. From (36) to (54), we obtain

$$
\frac{U^{\prime}(x)}{U(x)}=-2 \lambda x+\frac{\alpha}{x}+\frac{2 \beta x}{x^{2}-1}
$$

Hence,

$$
U(x)=|x|^{\alpha}\left|x^{2}-1\right|^{\beta} e^{-\lambda x^{2}},
$$

is the solution in $]-\infty,+\infty[$ and $[-1,1]$.

If $\beta \neq 0$ and $\alpha, \beta>-1,(8)$ and (49) hold in the following situations: 
$s_{1}:$ If $\lambda>0$, then

$$
\langle u, f\rangle=\int_{-\infty}^{+\infty}|x|^{\alpha}\left|x^{2}-1\right|^{\beta} e^{-\lambda x^{2}}\left(A \chi_{[-1,1]}(x)+B \chi_{]-\infty,+\infty[}(x)\right) f(x) d x .
$$

$s_{2}:$ If $\lambda<0$, then

$$
\langle u, f\rangle=A_{1} \int_{-1}^{1}|x|^{\alpha}\left(1-x^{2}\right)^{\beta} e^{-\lambda x^{2}} f(x) d x
$$

\section{Particular case:}

If $\alpha=\beta=0$, then (36) becomes

$$
\left\{\begin{array}{l}
\left(x^{2}\left(x^{2}-1\right) u\right)^{\prime}+2 x\left(\lambda x^{4}-(\lambda+2) x^{2}+1\right) u=0, \\
\left\{2 \lambda(u)_{4}-(2 \lambda+3)(u)_{2}+1\right\} \times\left\{2 \lambda(u)_{4}-3(u)_{2}\right\} \neq 0 .
\end{array}\right.
$$

Thus,

$$
u=(1-k) \tilde{v}+k \delta_{0},
$$

where $\tilde{v}$ is symmetric semi-classical form of class two satisfies (see $[8,10])$

$$
\left(\left(x^{2}-1\right) \tilde{v}\right)^{\prime}+2 x\left(\lambda x^{2}-\lambda-1\right) \tilde{v}=0 .
$$

Notice that the linear functional $u$ defined by (116) is regular if and only if $k$ satisfies[11]

$$
1+k \sum_{m=0}^{n} \frac{P_{m}^{2}(0)}{\left\langle v, P_{m}^{2}\right\rangle} \neq 0, n \geq 0
$$

where $\left\{P_{n}\right\}_{n \geq 0}$ is the MOPS with respect to $\tilde{v}$.

In this case

$$
\langle u, f\rangle=\frac{1-k}{\sqrt{\lambda \pi}} \int_{-\infty}^{+\infty} e^{-\lambda x^{2}} f(x) d x+k f(0), \lambda>0 .
$$

$\mathbf{B}_{3}$. From (38) to (54) we get

$$
\frac{U^{\prime}(x)}{U(x)}=-2 \lambda x+\frac{2 \alpha x}{x^{2}-1}-\frac{2 \beta x}{\left(x^{2}-1\right)^{2}}
$$

therefore

$$
U(x)=\left|x^{2}-1\right|^{\alpha} e^{-\lambda x^{2}-\frac{\beta}{1-x^{2}}},
$$

is the solution in $]-\infty,+\infty[$ and $[-1,1]$.

If $\beta \neq 0$ and $\alpha>-1$, then (8) and (49) hold in the following situations:

$s_{1}:$ If $\lambda>0$ and $\beta>0$, then

$$
\langle u, f\rangle=\int_{-\infty}^{+\infty}\left|x^{2}-1\right|^{\alpha} e^{-\lambda x^{2}-\frac{\beta}{1-x^{2}}}\left(A \chi_{[-1,1]}(x)+B \chi_{]-\infty,+\infty[}(x)\right) f(x) d x .
$$

$s_{2}:$ If $\lambda<0$ and $\beta>0$, then

$$
\langle u, f\rangle=A_{1} \int_{-1}^{1}\left(1-x^{2}\right)^{\alpha} e^{-\lambda x^{2}-\frac{\beta}{1-x^{2}}} f(x) d x .
$$

\section{Particular CASE:}

If $\beta=0$ and $\alpha=2$, then from (38) we obtain

$$
\left\{\begin{array}{l}
\left(\left(x^{2}-1\right)^{2} u\right)^{\prime}+2 x\left(\lambda x^{4}-2(\lambda+2) x^{2}+\lambda+4\right) u=0 \\
2 \lambda(u)_{4}-(2 \lambda+7)(u)_{2}+1 \neq 0
\end{array}\right.
$$


Then,

$$
u=(1-2 k) h_{\sqrt{\lambda^{-1}}} \mathcal{H}+k\left(\delta_{1}+\delta_{-1}\right),
$$

where $\mathcal{H}$ is the Hermite linear functional that satisfies[7]

$$
\mathcal{H}^{\prime}+2 x \mathcal{H}=0
$$

The above linear functional $u$ is regular if and only if $k$ satisfies (see [2])

$$
\left|\begin{array}{cc}
1+k K_{n}(1,1) & k K_{n}(1,-1) \\
k K_{n}(1,-1) & 1+k K_{n}(-1,-1)
\end{array}\right| \neq 0, n \geq 0 .
$$

From (120), applying both linear functionals $x^{2 n}$ we get

$$
(u)_{2 n}=(1-2 k) \frac{\lambda^{-n} \Gamma(2 n+1)}{2^{2 n} \Gamma(n+1)}+2 k, \quad n \geq 0 .
$$

Finally, from (120) we obtain

$$
\langle u, f\rangle=\frac{1-2 k}{\sqrt{\lambda \pi}} \int_{-\infty}^{+\infty} e^{-\lambda x^{2}} f(x) d x+k(f(1)+f(-1)), \lambda>0 .
$$

$\mathbf{B}_{4}$. From (40) to (54), we have

$$
\frac{U^{\prime}(x)}{U(x)}=-2 x+\frac{2 \alpha}{x}+\frac{2 \beta}{x^{3}}
$$

Thus,

$$
U(x)=|x|^{2 \alpha} e^{-x^{2}-\frac{\beta}{x^{2}}}
$$

is the solution in $]-\infty,+\infty[$.

If $\beta \geq 0$ and $\alpha>-\frac{1}{2}$, then (8) and (49) hold.

Thus

$$
\langle u, f\rangle=k \int_{-\infty}^{+\infty} f(x)|x|^{2 \alpha} e^{-x^{2}-\frac{\beta}{x^{2}}} d x .
$$

Remark 7. If $\beta=0$, then the form defined by (122) is the generalized Hermite polynomials [7].

$\mathbf{B}_{5.1}$. From (43) to (54), we get

$$
\frac{U^{\prime}(x)}{U(x)}=-4 \lambda x^{3}++2 \alpha x+\frac{2 \beta x}{x^{2}-1}
$$

So

$$
U(x)=\left|x^{2}-1\right|^{\beta} e^{-\lambda x^{4}+\alpha x^{2}},
$$

is the solution in $\mathbb{R}$ and $[-1,1]$.

If $\beta \neq 0$ and $\beta>-1,(8)$ and (49) hold in the following situations:

$s_{1}:$ If $\lambda>0$, then

$$
\langle u, f\rangle=\int_{-\infty}^{+\infty}\left|x^{2}-1\right|^{\beta} e^{-\lambda x^{4}+\alpha x^{2}}\left(A \chi_{[-1,1]}(x)+B \chi_{]-\infty,+\infty[}(x)\right) f(x) d x .
$$

$s_{2}:$ If $\lambda<0$, then

$$
\langle u, f\rangle=A_{1} \int_{-1}^{1}\left(1-x^{2}\right)^{\beta} e^{-\lambda x^{4}+\alpha x^{2}} f(x) d x .
$$

$\mathbf{B}_{5.2}$. From (45) to (54), we obtain

$$
\frac{U^{\prime}(x)}{U(x)}=-4 x^{3}+2 \beta x+\frac{\alpha}{x}
$$


Hence,

$$
U(x)=|x|^{\alpha} e^{-x^{4}+\beta x^{2}},
$$

is the solution in $\mathbb{R}$.

Furthermore, for $\alpha>-1$, then (8) and (49) hold.

Thus,

$$
\langle u, f\rangle=A \int_{-\infty}^{+\infty} f(x)|x|^{\alpha} e^{-x^{4}+\beta x^{2}} d x .
$$

Particular case:

If $\alpha=0$, then (45) becomes

$$
\left\{\begin{array}{l}
\left(x^{2} u\right)^{\prime}+2 x\left(2 x^{4}-\beta x^{2}-1\right) u=0, \\
4 \lambda(u)_{4}-2 \beta(u)_{2}-1 \neq 0 .
\end{array}\right.
$$

Thus,

$$
u=(1-k) \breve{v}+k \delta_{0},
$$

where $\breve{v}$ is symmetric semi-classical form of class two satisfies (see $[8,10]$ )

$$
\breve{v}^{\prime}+2 x\left(2 x^{2}-\beta\right) \breve{v}=0 .
$$

In this case

$$
\langle u, f\rangle=(1-k) \mathcal{A} \int_{-\infty}^{+\infty} e^{-x^{4}+\beta x^{2}} f(x) d x+k f(0),
$$

where $\mathcal{A}=\left(\int_{-\infty}^{+\infty} e^{-x^{4}+\beta x^{2}} d x\right)^{-1}$.

$\mathbf{B}_{6}$. From (47) to (54), we get

$$
\frac{U^{\prime}(x)}{U(x)}=-6 x^{5}-4 \alpha x^{3}-2 \beta x
$$

Thus,

$$
U(x)=e^{-x^{6}-\alpha x^{4}-\beta x^{2}}
$$

is the solution in $\mathbb{R}$. As a consequence $u$ is represented by

$$
\langle u, f\rangle=k \int_{-\infty}^{+\infty} f(x) e^{-x^{6}-\alpha x^{4}-\beta x^{2}} d x .
$$

This is an example of Freud linear functional (see [8] and [16]).

\section{References}

[1] J. Alaya and P. Maroni, Symmetric Laguerre-Hahn forms of class $s=1$, Integral Transforms Spec. Funct. 4 (1996), 301-320. https://doi.org/10.1080/10652469608819117

[2] R. Álvarez-Nodarse, J. Arvesú and F. Marcellán, Modifications of quasi-definite linear functionals via addition of delta and derivatives of delta Dirac functions, Indag. Math. (N. S.) 15 (2004), 1-20. https://doi.org/10.1016/s0019-3577(04)90001-8

[3] M.J. Atia, Some symmetric sem-classical orthogonal polynomials of class $0 \leq s \leq 5$. Integral Transforms Spec. Funct. 17(7) (2006), 469-483. https://doi.org/10.1080/10652460600725085

[4] J. Arvesú, M. J. Atia, and F. Marcellán, On semiclassical linear functionals: The symmetric companion, Commun. Anal. Theory Contin. Fract. 10 (2002), 13-29. 
[5] S. Belmehdi, On semi-classical linear functionals of class $s=1$. Classification and integral representations, Indag. Math. (N. S.), 3 (1992), 253-275. https://doi.org/10.1016/0019-3577(92)90035-j

[6] A. Branquinho and F. Marcellán, Generating new classes of orthogonal polynomials, Int. J. Math. Math. Sci, 19 (1996), 643-656. https://doi.org/10.1155/s0161171296000919

[7] T. S. Chihara, An introduction to orthogonal polynomials, Gordon and Breach, New York. 1978.

[8] A. M. Delgado and F. Marcellán, Semi-classical linear functionals of class 2. The symmetric case, In Difference Equations, Special Functions, and Orthogonal Polynomials. Proceedings of the International Conference Munich 2005, S. Elaydi et al. Editors. World Scientific, Singapore. 2007. 122-130.

[9] F. Marcellán, M. Sghaier, and M. Zaatra, Semi-classical linear functionals of class three. The symmetric case. J. Difference Equ. Appl. 19(1) (2013), 162-178. https://doi.org/10.1080/10236198.2012.712969

[10] F. Marcellán, M. Sghaier, and M. Zaatra, On semiclassical linear functionals of class $s=2$. Classification and Integral representations, J. Difference Equ. Appl. 18(6)(2012), 973-1000. https://doi.org/10.1080/10236198.2010.540241

[11] F. Marcellán, and P. Maroni, Sur l'adjonction d'une masse de Dirac à une forme régulière et semi-classique. Ann. Mat. Pura ed Appl. 162 (IV) (1992), 1-22. https://doi.org/10.1007/bf01759996

[12] P. Maroni, On a regular form defined by a pseudo-function.Numer. Algorithms, 11 (1996), 243-254.

[13] P. Maroni, An integral representation for Bessel form, J. Comput. Appl. Math., 57 (1995), $251-260$. https://doi.org/10.1016/0377-0427(93)e0249-1

[14] P. Maroni, Sur la décomposition quadratique d'une suite de polynômes orthogonaux, I, Riv. Mat. Pura Appl., 6 (1991), 19-53.

[15] P. Maroni, Une théorie algébrique des polynômes orthogonaux. Application aux polynômes orthogonaux semiclassiques, Orthogonal Polynomials and their applications, C. Brezinski et al Editors. IMACS Ann. Comput. Appl. Math. Baltzer, Basel. 9 (1991). 95-130.

[16] P. Nevai, Orthogonal polynomials associated with $\exp \left(-x^{4}\right)$, Proc. Canad. Math. Soc. 3(1983), $263-285$.

[17] M. Sghaier and J. Alaya, Semiclassical forms of class $s=2$ : the symmetric case, when $\Phi(0)=0$, Methods Appl. Anal., 13(4) (2006), 387-410. https://doi.org/10.4310/maa.2006.v13.n4.a5

[18] J. Shohat, A differential equation for orthogonal polynomials, Duke Math. J., 5 (1939), $401-417$. https://doi.org/10.1215/s0012-7094-39-00534-x 